\section{Drug discovery in China}

Organizations worldwide have shown growing interest in China as a location for drug discovery and development. This month, we feature two people closely involved in harnessing the therapeutic potential of compounds discovered in China.

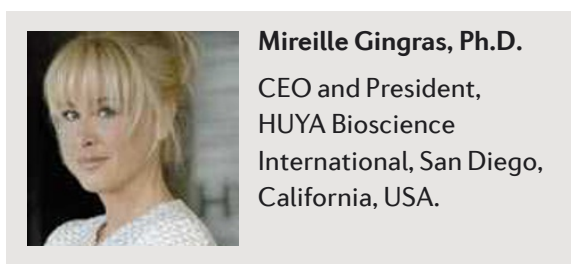

Chinese companies providing contract research services for drug discovery and development have rapidly established themselves in recent years. There is also increasing interest in the potential of China as a source for novel preclinical and clinical compounds, says Mireille Gingras, CEO and President of HUYA Bioscience International, a company that has pioneered a novel co-development model in which such compounds are identified and licensed for development in Western countries.

"Through our partnerships with Chinese companies and institutes, we can use preclinical and clinical data generated in China to inform the drug development process in the West," Gingras says. "Although additional clinical trials must be done in the West, the Chinese data are helpful in lowering the risk and enhancing the efficiency of drug development. In this way, pharmaceutical companies have the benefit of access to novel validated compounds, and Chinese organizations have the benefit of gaining access to Western markets for their compounds."

Gingras became interested in drug discovery in China while working as a licensing consultant searching for novel early stage compounds throughout Europe and Asia. "Many of us were looking in the same places, and the pools of novel compounds were depleted," she says. "I then spent time in China meeting with heads of government research institutions, biotechnology parks, incubators and pharmaceutical companies, and I formed HUYA because I recognized that there were significant opportunities for drug discovery and development. We currently have licensed two compounds from China that are in preclinical development in the United States, thus validating our model."

The key steps in achieving this success were her education, broad multicultural business experience and investing the necessary time to build relationships, feels Gingras. "First, my scientific background, including a Ph.D. and two postdocs in neurobiology, was a fundamental

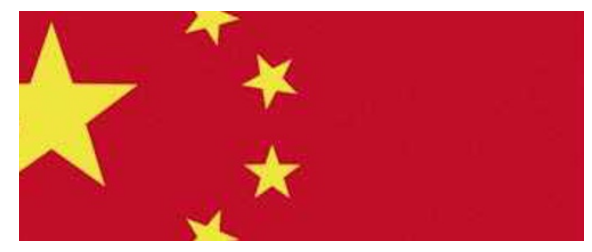

step," she says. "Second, having started and grown several companies, such as MIR3, a software company that developed advanced on-line treatment programs for neuroaddictive diseases, I built the operational experience needed for my current position." Her work in countries in Europe, North America and Asia also gave her valuable experience in collaborating with individuals from many cultures. "The third step leading to my current position as CEO was spending most of the past 3 years in China, which has allowed me to successfully establish and build relationships there," she concludes.

Building on these relationships to create the partnerships on which the company is based has been challenging at times. "We're a young, flexible company that is willing to take risks, and can work very quickly to in-license compounds from China. However, l've had to learn to be patient when partnerships, negotiations and agreements take longer than I expected because of bureaucracy in larger companies," says Gingras. Nevertheless, this aspect is also the part of her role that she finds most rewarding: "Every day we break geographical and cultural barriers, and I am thrilled when people understand what we are doing and want to be involved."

Wang Xiaoling,
M.D., Ph.D.
$\begin{aligned} & \text { Director of the Institute } \\ & \text { of Materia Medica, } \\ & \text { Beijing, China. }\end{aligned}$

Fifty years ago, the Institute of Materia Medica (IMM), Beijing, was established as one of the main Chinese institutes for drug research, and has since developed more than 100 drugs used for treating diseases including cancer, hepatitis and cardiovascular disease, primarily in China. Today, the priority of the IMM is the discovery and development of novel compounds from natural products to treat commonly occurring diseases both in China and internationally, with a growing emphasis on the global market, says Wang Xiaoling, Director of the IMM.

"Owing to the explosive growth in Chinese pharmaceutical development, our institutions are able to work closely with large international pharmaceutical companies and smaller companies such as HUYA Bioscience International," he explains. "These collaborations provide our institutions with good opportunities to understand US standards and regulations, and give us the potential to adapt to the Western standard of drug development."

Wang's interest in drug discovery and his association with the IMM stems from his educational background as an M.D. and a Ph.D. in pharmacology. He was attracted to medicine early in his career, and following an education that was interrupted by the Cultural Revolution, he took the opportunity to study at the Peking Medical School in 1975.

After gaining his medical qualifications, and a master's degree from Peking Union Medical College, he chose to go to Germany to study for a year. "Going to another country to be exposed to technologies and innovations and new scientific concepts was very valuable, especially in contrast to what China had to offer in the 1970s and 1980s," he says. His professors encouraged him to stay, and he subsequently completed a Ph.D. in pharmacology and electrophysiology.

In 1988, Wang returned to China to pursue his career in life sciences, and progressed to become Director of the IMM in 1997. Now, as well as continuing to be actively involved in research, he oversees the activities of the Institute. This includes more than 100 professors and associate professors across seven departments, with expertise in natural products research, computer-aided drug design, combinatorial chemistry, molecular pharmacology, drug analysis and high-throughput screening. In the past decade in this role, the situation at the IMM has changed significantly and continues to evolve. "First, our budgets are much more robust in comparison to a few years ago," Wang says. "Second, it is clear that drug research and development will be shifted to some extent from our national institutions and universities to the pharmaceutical industry."

In this respect, the relationships being developed with Western companies are important, and will provide a new angle on future drug development at the Institute, says Wang. This opportunity to learn from others' perspectives is also reflected in what he feels have been some of the most valuable experiences in his career so far. "I would encourage all scientists and students to explore other cultures and thoughts - it can be very rewarding," he says.

WEB SITE

Career snapshots: http://www.nature.com/naturejobs/ magazine/career snaps.html 